\title{
Eine neue fluorometrische Methode zur Aldosteron-Bestimmung im Harn
}

\author{
Von E. Nowotny ${ }^{1}$ ) und HJ. Staudinger \\ Aus dem Physiologisch-Chemischen Institut der Universität Gießen (Direktor: Prof. Dr. Hj. Staudinger)
}

(Eingegangen am 16. November 1965)

\begin{abstract}
Es wird eine neue Methode für die Bestimmung von Aldosteron im menschlichen Harn beschrieben. Durch die empfindliche Nachweisreaktion auf Lithiumhydroxyd benötigt man nur $1 / 10$ Volumen Tagesharn. Die Methode ist genügend richtig, genau und spezifisch und wegen ihrer Einfachheit als Routineverfahren geeignet. Die Ergebnisse bei 20 Normalpersonen und bei Patienten wurden mitgeteilt und mit den Ergebnissen anderex Autoren verglichen.

A new method is reported for the determination of aldosterone in human urine. Only $1 / 10$ of the daily urine volume is required for the sensitive test reaction on lithium hydroxide. The accuracy and specificity of the method are satisfactory and, owing to its simplicity, it is suitable as a routine procedure. The results are given for 50 normal people and for patients and compared wirth the results of other authors.
\end{abstract}

Es gibt eine Reihe von Methoden zur Aldosteron-Bestimmung, die sich gut bewährt haben, da sie spezifisch und empfindlich sind. Sie sind aber mit einem hohen Arbeitsaufwand verbunden (1-16). Daher entschlossen wir uns, in Anlehnung an eine in unserem Institut früher ausgearbeitete Methode (17), eine einfache AldosteronBestimmung für den Routinegebrauch zu entwickeln. Die Bestimmung ist mit $1 / 10$ Vol. des 24-Stdn.-Harns möglich; sie ist dabei einfacher als andere mit kleinen Harnvolumen arbeitende chemische Methoden (GERDES und Starb [16], KLIMAN und Mitarbeiter [7]). Nach einer Vorreinigung an einer Kieselgelsäule wird das Steroidgemisch durch zweidimensionale Dünnschichtchromatographie getrennt (17). Für den quantitativen Nachweis benutzten wir die Fluoreszenzmessung auf Lithiumhydroxyd-Preßlingen (18). Aldosteron gibt wie andere $\triangle^{4}$-3-Ketosteroide auf der Oberfläche eines Lithiumhydroxyd-Preßlings eine empfindliche und für diese Steroide spezifische Fluoreszenz (19). Die Spezifität, die Genauigkeit und die Richtigkeit der im folgenden genau beschriebenen Methode sind für den klinischen Gebrauch ausreichend.

\section{Methodik}

\section{Reagenzien}

Chloroform: 2 / Chloroform werden 5 Min. mit $200 \mathrm{~m} /$ 0,1-proz. Kaliumpermanganat-Lösg. geschüttelt und dreimal mit Wasser gewaschen. Nach 24-stdg. Trocknen über wasserfreiem Kaliumcarbonat wird im Dunkeln über eine Kolonne destilliert. Für die Chromatographie wird Chloroform der Qualität „Uvasol“ (Fa. Merck) verwendet.

Methylenchlorid: wird wie Chloroform gereinigt.

Aceton: „Uvasol" (Fa. Merck).

Acetatpuffer $\mathrm{pH}$ 4,5:3,8 $\mathrm{g}$ wasserfreies Natriumacetat und 2,5 $\mathrm{ml}$ Essigsäure werden mit dest. Wasser auf $1 l$ aufgefüllt.

Äthanol: abs., p. a. (Fa. Riedel-de Haën) wird über eine RaschigKolonne einmal fraktioniert.

Methanol: p. a. (Fa. Riedel-de Haën) wird wie Äthanol gereinigt. Kieselgel: unter 0,08 mm „Merck" für Säulenchromatographie.

Kieselgel: $\mathrm{HF}_{254}$, "Merck “ für Dünnschichtchromatographie.

Die verwendeten Glasgeräte werden zunächst mit einer 2-proz. wäßr. Lösung von „RBS 25“ (Fa. Carl Roth, Karlstuhe) und dann mit Chromschwefelsäure gereinigt.

\section{Extraktion des Harns}

Falls der Harn nicht frisch untersucht werden kann, wird ex bei $-20^{\circ}$ eingefroren. Alle folgenden Arbeitsgänge sind grundsätz-

1) Stipendiat der Alexander-von-Humboldt-Stiftung. lich in diffusem Tageslicht bzw. bei gedämpftem künstlichem Licht durchzuführen, da sonst beträchtliche Aldosteronverluste eintreten können. Ein Zehntel der Tagesharnmenge wird mit konz. Salzsäure auf pH 1 cingestellt, 2 Stdn. bei Raumtemperatur stehengelassen und dann mit Natriumchlorid gesättigt. Dann schüttelt man viermal je $5 \mathrm{Min}$. (evtl. mit einer Schüittelmaschine) mit Methylenchlorid aus (einmal mit 1/3, dreimal mit je $1 / 1$ des eingesetzten Harnvolumens). Die Methylenchloridlösung wird danach durch Zentrifugieren geklärt. Je nach der mit Methylenchlorid extrahierten Menge Harnpigmente wäscht man die vereinigten Methylenchloridextrakte ein- oder zweimal mit $1 /-k$ Volumen $0,5 \mathrm{~N} \mathrm{NaOH}$ und zweimal mit $1 / 4$ Volumen Wasser. Die vereinigten Waschflüssigkeiten werden mit $30 \mathrm{ml}$ Methylenchlorid nachextrahiert. Die vereinigten Methylenchloridlösungen dampft man nach Trocknen über wasserfreiem Natriumsulfat bei $35^{\circ}$ im Vakuum in einem Rotationsverdampfer ein; durch die Kapillare leitet man einen schwachen Stickstoffstrom. Alle im folgenden erwähnten Destillationen werden in der gleichen Weise durchgeführt. (Bei stark lipoidhaltigem Harn [Nephrose] ist es nötig, den Verdampfungsrückstand in $5 \mathrm{~m} l$ Aceton aufzunehmen, zu filtrieren und die Lösung erneut einzudampfen; bei normalen Harnen kann dieser Schritt entfallen.) Der Rückstand wird nacheinander mit $5 \mathrm{~m} /$ und zweimal mit je $2,5 \mathrm{~m} /$ Acetatpuffer $\mathrm{pH} 4,5$ versetzt und mit einem Glasstạb gut durchgearbeitet, um das Aldosteron vollständig aus dem „,fetten“ Verdampfungstückstand zu lösen. Die vereinigten wäßr. Lösungen extrahiert man zweimal mit je $30 \mathrm{ml}$ Methylenchlorid, trocknet über wasserfreiem Natriumsulfat und verdampft das Lösungsmittel.

\section{Säulenchromatographische Vorreinigung}

Wir arbeiteten in Anlehnung an das Verfahren ron NEHER und WETTSTEIN (1).

Man gibt in ein Chromatographierohr $(32 \times 1,6 \mathrm{~cm})$, das unten mit einem Wattestopfen verschlossen ist, eine Aufschlämmung von $1 \mathrm{~g}$ Kieselgel in Chloroform. Wenn das Chloroform fast durchgesickert ist, bringt man den Verdampfungsrückstand des Methylenchlorid-Extraktes quantitativ mit dreimal je $1 \mathrm{~m} /$ Chloroform auf die Säule, eluiert mit $40 \mathrm{ml}$ Chloroform/Aceton (99:1) die störenden Pigmente und mit $70 \mathrm{~m} / \mathrm{Chloroform} /$ Aceton $(50: 50)$ und danach mit $10 \mathrm{~m} l \AA \AA$ thanol die Steroide. Das steroidhaltige Eluat engt man ein, den Rückstand überführt man quantitativ mit Chlotoform in ein Spitzröhrchen und dampft die Lösung ein.

\section{Dïnnscbichtchromatographie}

Sie erfolgte in Anlehnung an Nishikaze und Staudinger (17). Die Glasplatten $(20 \times 20 \mathrm{~cm})$ werden mit Kieselgel $\mathrm{HF}_{254}(25 \mathrm{~g}$ in $71 \mathrm{~m} /$ Wasser für 5 Platten, Schichtdicke $200 \mu$ ) beschichtet, 15 Min. an der Luft und über Nacht bei $100^{\circ}$ getrocknet. Dann bewahrt man sie in einem Exsikkator über Blaugel auf. Den Startpunkt markiert man $3 \mathrm{~cm}$ von der linken und $2,5 \mathrm{~cm}$ von der unteren Plattenkante entfernt. Die Laufstrecke beträgt nach beiden Richtungen $15 \mathrm{~cm}$.

Der Rückstand im Spitzröhrchen wird in $0,1 \mathrm{~m} /$ Chloroform aufgenommen und quantitativ unter Ausschluß des Tageslichtes 
punktförmig auf den Startpunkt der Platte aufgetragen. Das Röhrchen spült man noch zweimal mit je $0,1 \mathrm{~m} /$ Chloroform aus und trägt auch dicse Lösungen auf die Platte auf. Zum Schluß trägt man noch je $10 \mu$ g der Leitsteroide (17) auf den gleichen Fleck auf und chromatographiert zweidimensional im Dunkeln (1. Cyclohexan/ Isopropanol [7:3]; 2. Chloroform/Eisessig [8:2]).

Der Aldosteronfleck wird mit Hilfe der Leitsteroide im kurzwelligen UV-Licht $(254 \mathrm{~m} \mu)$ lokalisiert, von der Platte gekratzt und mit $2 \mathrm{~m} /$ Methanol eluiert. Nach Zentrifugieren pipettiert man $1,5 \mathrm{~m} /$ Überstand in ein Spitzröhrchen, versetzt den Rückstand mit weiteren $2 \mathrm{~m} /$ Methanol, zentrifugiert und überführt weitere $1,5 \mathrm{~m} /$ in das Schliffröhrchen. Der Rückstand wird nochmal mit $2 \mathrm{ml} \mathrm{Me}$ thanol versetzt. Nach Abzentrifugieren entnimmt man $2 \mathrm{~m} l$. und vereinigt sie mit den anderen Eluaten. Man verdampft einen aliquoten Tcil dieșer Lösung $(0,1-0,3 \mathrm{~m} /$, je nach Aldosterongehalt) für die Fluoreszenzreaktion. Zur Ermittlung des Plattenleerwertes kratzt man einen gleichgroßen Fleck von einer Stelle der gleichen Platte, die im UV-Licht $(254 \mathrm{~m} \mu)$ keine Absorption gibt, eluiert das Kieselgel in gleicher Weise mit Methanol und verdampft das gleiche Volumen des methanolischen Eluates wie beim Hauptwert.

\section{Quantitative Bestimnung}

Hauptjvert (H): Man löst den Verdampfungsrückstand; der das Aldosteron enthält, in $0,1 \mathrm{~m} l$ reinstem Chloroform auf, engt die Lösung im Stickstoffstrom bei Raumtemperatur auf etwa $0,01 \mathrm{~m} /$ ein und trägt diese Lösung, wie bereits von uns beschrieben (18), auf einen Lithiumhydroxyd-Preßling auf. Das Spitzröhrchen wird noch zweimal mit je $0,1 \mathrm{~m} /$ Chloroform ausgespült, und die Spüllösungen werden nach Einengen auf $0,01 \mathrm{ml}$ auf den gleichen Preßling aufgetragen.

Standardwert (S): Als Vergleich werden $0,1 \mu \mathrm{g}$ in Chloroform gelöstes Aldosteron eingedampft, in reinstem Chloroform aufgenommen und unter den gleichen Bedingungen wie beim Hauptwert aufgetragen.

Leervert $(L)$ : Die gleiche Menge Methanol wie beim Hauptwert wird im Spitzröhrchen im Stickstoffstrom verdampft und der Rückstand in gleicher Weise wie beim Hauptwert in Chloroform aufgenommen und auf einen Preßling aufgetragen.

Plattenleervert $(P)$ : Der Verdampfungstückstand des Eluates wird mit der gleichen Menge Chloroform wie beim Hauptwert behandelt und die Lösungen werden auf einen Preßling aufgetragen.

Es ist $\mathrm{zu}$ beachten, daß auf alle 4 Preßlinge das gleiche Volumen Chloroform in der gleichen Zeit aufgetragen wird. Ferner wird empfohlen, von $\mathrm{H}, \mathrm{S}, \mathrm{L}$ und $\mathrm{P}$ jeweils Doppelwerte auf zwei Preßlingen anzufertigen. Vor dem Auftragen der Lösungen muß man die Preßlinge fluorometrisch auf Verunreinigungen prüfen und nur Preßlinge mit annähernd gleicher, niedriger Grundfluoreszenz aussuchen ${ }^{1}$ ). Nach dem Auftragen der Chloroformlösungen werden die Tabletten fluorometrisch gemessen. Dann werden sie 20 Min. auf $100^{\circ}$ erwärmt und nach Abkühlen erneut gemessen. Das Vorgehen und die Meßanordnung sịnd in unserer früheren Arbeit (18) genau beschrieben. Auch die hochgereinigten Lösungsmittel und die anderen verwendeten Chemikalien enthalten stets eine kleine Menge fluoreszierender Begleitstoffe, die mitgeschleift werden; deshalb sind die verschiedenen Korrekturen nötig. Die Fluoreszenzintensitäten der Preßlingc, auf die der Hauptwert $(\mathrm{H})$ und der Plattenleerwert $(P)$ aufgetragen werden, werden vor und nach dem Erhitzen gemessen. Die wahre Intensität der Fluoreszenz des Harnaldosterons (Ald) ergibt sich aus folgender Rechnung:

$$
\begin{aligned}
& F_{H \text { korr. }}=F_{H(\text { nach })}-F_{H(\text { vor })} \quad F=\text { relative Intensität der } \\
& \text { Fluoreszenz } \\
& F_{P \text { korr. }}=F_{P(\text { nach })}-F_{P(\text { ror })} \quad \text { (vor) }=\text { vor dem Erhitzen des } \\
& \text { Preßlings } \\
& F_{\text {Ald }}=F_{\text {H korr. }}-F_{P} \text { korr. } \quad(\text { nach })=\text { mach dem Erhitzen } \\
& \text { des Preßlings } \\
& \mathrm{H}=\text { Hauptwert } \\
& \mathrm{P}=\text { Plattenleerwert } \\
& \text { Ald }=\text { Aldosteron }
\end{aligned}
$$

1) Es ist zweckmäßig, sich einen größeren Vorrat ausgesuchter Preßlinge anzufertigen.
Der Fluoreszenzwert für den Aldosteron-Standard (S) muß in folgender Weise korrigiert werden:

$F_{S \text { korr. }}=F_{S(\text { nach })}-F_{S(\text { vor })} \quad S=$ Aldosteron-Standard

\section{Ergebnisse}

Spezifität der Methode

\section{Dïnnschichtchromatographie}

Wie Nishikaze und Staudinger (17) zeigen konnten, wandert Aldosteron bei zweidimensionaler Dünnschichtchromatographie (1. Cyclohexan/Isopropanol [7:3]; 2. Chloroform/Eisessig [8:2]) stets im gleichen Verhältnis zu 11-Dehydrocorticosteron (A) und 11-Desoxycortisol (S). Bei dieser zweidimensionalen Chromatographie erscheint das Aldosteron aus Harnextrakten als einheitlicher Fleck. Um seine Reinheit und Identität mit authentischem Aldosteron zu prüfen, haben wir diesen Aldosteronfleck ausgekratzt und in den Systemen Äthanol (95\%)/Chloroform (8:92) und Methylenchlorid/ Eisessig/Methanol (90:10:5) erneut zweidimensional rechromatographiert. Der Fleck erschien erneut einheitlich und rund, was dafür spricht, daß das Aldosteron bereits nach der ersten zweidimensionalen Chromatographie sauber von anderen Steroiden und Verunreinigungen abgetrennt worden ist. Die $R_{F-}$ Werte des aus Harn isolierten Aldosterons stimmen auch in diesem System mit denen von reinem Aldosteron überein.

\section{Fluoreszenz- und Absorptionsspektren der Schuefelsäure- chromogene}

Wie Abraham und Straudinger zeigten (20), geben die Corticosteroide beim Erhitzen mit konz. Schwefelsäure Fluoreszenzspektren mit jeweils spezifischen Maxima. Diese Eigenschaft haben wir zur Beurteilung der Einheitlichkeit des aus dem Harn gewonnenen Aldosterons herangezogen. Der nach zweidimensionaler Dünnschichtchromatographie isolierte Aldosteronfleck wurde ausgekratzt und das Fluoreszenzspekrum der Schwefelsäurrechromogene gemessen. Bei drei so untersuchten Harnen (Mischharne von verschiedenen Personen) war das Fluoreszenzspektrum identisch mit dem Spektrum von reinem Aldosteron, das zuvor im gleichen System chromatographiert wurde. Auch dieser Befund zeigt, daß das aus dem Harn isolierte Aldosteron weitgehend rein und mit authentischem Aldosteron identisch ist. Das Maximum des Fluoreszenzspektrums von reinem Aldosteron, das in dem angegebenen System chromatographiert wurde, weicht gegenüber reinem, ,nicht chromatographiertem" Aldosteron etwas ab (525 $\mathrm{m} \mu$ gegenüber $545 \mathrm{~m} \mu$ ). Auch das Absorptionsmaximum der Schwefelsäurechromogene von Harnaldosteron ist mit dem von authentischem Aldosteron identisch. Selbst wenn das durch zweidimensionale Dünnschichtchromatographie aus dem Harn isolierte Aldosteron noch nicht ganz rein sein sollte, wird die Spezifität der Methode nicht betroffen, da der anschließende Nachweis des Aldosterons, die Fluoreszenz auf der Lithiumhydroxyd-Oberfläche, spezifiscb für $\Delta^{4}$-3-Ketosteroide ist. Keines der bekannten $\Delta^{4}$-3-Ketosteroide wandert aber bei der zweidimensionalen Dünnschichtchromatographie zusammen mit 
Aldosteron. Die Spezifität dieser Methode beruht also auf dem angegebenen Trennverfahren und auf der für $\Delta^{4}$-3-Ketosteroide spezifischen und sehr empfindlichen Fluoreszenzreaktion auf Lithiumhydroxyd-Preßlingen.

\section{Richtigkeit (Wiederfindung)}

Zur Prüfung der Richtigkeit („accuracy“) der Methode wurde die Wiederfindung („,recovery") der einzelnen Schritte der Methode nach Zugabe verschiedener Mengen von Aldosteron geprüft:

Kieselgelsäule: Es wurden $1 \mu \mathrm{g}$ und $5 \mu \mathrm{g}$ Aldosteron auf die Säule gegeben. Die Wiederfindung war für beide Konzentrationen bei je 5 Bestimmungen durchschnittlich $91 \%$.

Dünnschicbtchromatograpbie: Es wurden $1 \mu \mathrm{g}, 3 \mu \mathrm{g}$ und $5 \mu \mathrm{g}$ Aldosteron auf Dünnschichtplatten aufgetragen und zweidimensional chromatographiert. Die durchschnittliche Wiederfindung bei 12 Versuchen betrug für die drei angegebenen Konzentrationen $97 \%$.

Gesamtmethode: Es wurden Mengen von $1 \mu \mathrm{g}$ und $5 \mu \mathrm{g}$ Aldosteron einem zuvor analysierten Harn zugesetzt. Die Wiederfindung ist in Tabelle 1 angegeben.

Genauigkeit

Zur Ermittlung der Genauigkeit („precision“) untersuchten wir die Streuung des'Einzelwertes, indem wir den gleichen Mischharn $7 \mathrm{mal}$ analysierten. Dabei wurde ein Durchschnittswert von $20,6 \mu \mathrm{g} / 1000 \mathrm{ml}$ mit einer Standardabweichung $S$ von $\pm 2,27 \mu \mathrm{g}( \pm 11 \%)$ erhalten (Tab. 2).

Anwendung der Methode

Normaler Harn

Durchschnittswerte (Normalwerte)

Die Ergebnisse der Bestimmungen an 20 Normalpersonen beiden Geschlechtes im Alter von 18 bis 69 Jahren sind in Tabelle 3 zusammengefaßt. Die Normalausscheidung für die Gesamtpopulation ( $\bar{x} \pm 2$ s) liegt demnach zwischen 2 und $23 \mu \mathrm{g}$ pro Tag. Die mittlere Ausscheidung beträgt $12,4 \pm 5 \mu \mathrm{g}$ mit einer Schwankungsbreite in unserem Kollektiv von 3,65 und 20,6 $\mu \mathrm{g}$. Da es zwischen der Gruppe der Männer und der dẹ Frauen keinen signifikanten Unterschied gibt, eine Feststellung, die auch andere Autoren gemacht haben $(8,13,15)$, haben wir sie für die Bestimmung der Normalwerte zusammengefaßt.

Verlaufskurven (Individualschwankung)

Die Aldosteronausscheidung wurde bei einer normalen männlichen ( $36 \mathrm{~J}$.) und einer normalen weiblichen Person (40 J.) über einen Monat verfolgt. Die Ergebnisse sind in Abbildung 1 gezeigt. Daraus wird ersichtlich, daß bei der männlichen Versuchsperson die ausgeschiedenen Aldosteronmengen konstant sind ( $\pm 34 \%$ Abweichung vom Mittelwert). Die Aldosteronausscheidung bei der Frau hängt offerbar mit dem weiblichen Zyklus zusammen. Nach der Menstruation sind die Aldosteronwerte im Harn normal, in der Mitte des Zyklus fallen sie $a b$, in der $z$ weiten Hälfte steigen sie an und erreichen ver-
Tab. 1

Richtigkeit der Methode

Ergebnis von Wiederfindungsversuchen mit verschiedenen dem Harnextrakt zugegebenen Aldosteron-Mengen

\begin{tabular}{|c|c|c|c|}
\hline \multicolumn{2}{|c|}{ Aldostcron } & \multicolumn{2}{|c|}{$\begin{array}{c}\text { Prozentuale Wiederfindung } \\
\text { bei Zusatz von }\end{array}$} \\
\hline $\begin{array}{l}\text { zugesetzt } \\
\qquad \mu \mathrm{g}\end{array}$ & $\begin{array}{l}\text { wiedergef. } \\
\qquad \mu \mathrm{g}\end{array}$ & & \\
\hline 1 & 0,6 & 60 & - \\
\hline 1 & 0,8 & 80 & - \\
\hline 1 & 1,0 & 100 & - \\
\hline 5 & 3,1 & - & 62 \\
\hline 5 & 3,8 & - & 76 \\
\hline 5 & 4,8 & - & 96 \\
\hline 5 & 3,3 & 一 & 66 \\
\hline 5 & 4,6 & - & 92 \\
\hline \multirow[t]{2}{*}{5} & 4,6 & - & 92 \\
\hline & Durchs & $80 \%$ & $80 \%$ \\
\hline
\end{tabular}

Tab. 2

Genauigkeit der Methode

Mchrfachbestimmung bei cinem Mischharn

Aldosteronkonzentration im Harn $\mu \mathrm{g} / 1000 \mathrm{ml}$

$\begin{array}{r}20,0 \\ 21,8 \\ 18,1 \\ 21,8 \\ 21,8 \\ 23,8 \\ 16,9 \\ \hline \text { Durchschnitt } \overline{\mathbf{x}}: 20,6 \\ \mathrm{~s}: \pm 2,3 \\ \hline\end{array}$

Tab. 3

Ergebnisse der Aldosteronbestimmungen im Harn von gesunden Personen beiden Geschlechtes im Alter von 18 bis 69 Jahren

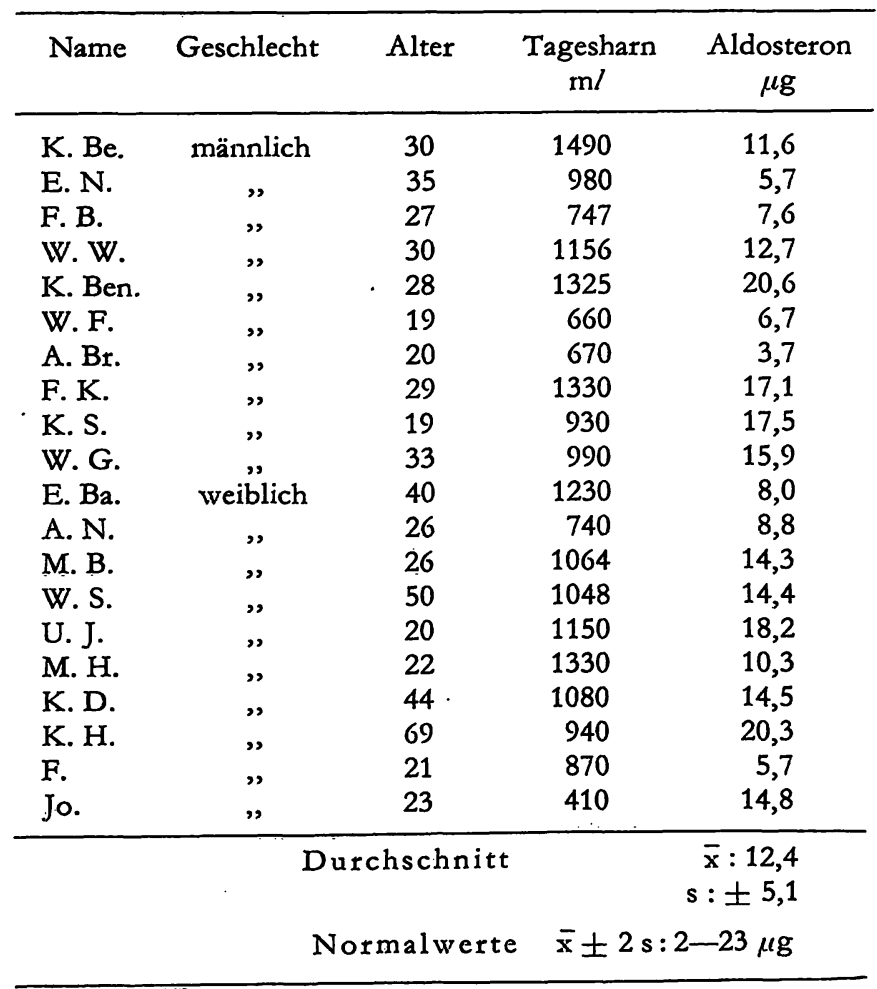


mutlich vor Anfang der Menses den höchsten Wert, der deutlich über der oberen Normalgrenze liegt. Die angegebenen Werte sind Doppelbestimmungen. - Gleichzeitig mit den exhöhten Aldosteronwerten wurde eine Neigung zur Wasserretention beobachtet. Interessant ist auch die.Feststellung, daß ein Anstieg der Aldosteronausscheidung im Harn mit einer Erhöhung der Progesteronkonzentration einhergeht (luteotrophische Phase). Außerdem ist erwiesen, daß das Aldosteron genau wie das Progesteron während der Schwangerschaft vermehrt gebildet wird und dann nach der Geburt wieder absinkt. Dies deutet auf eine Interrelation dieser beiden Hormone hin $(21,22)$.

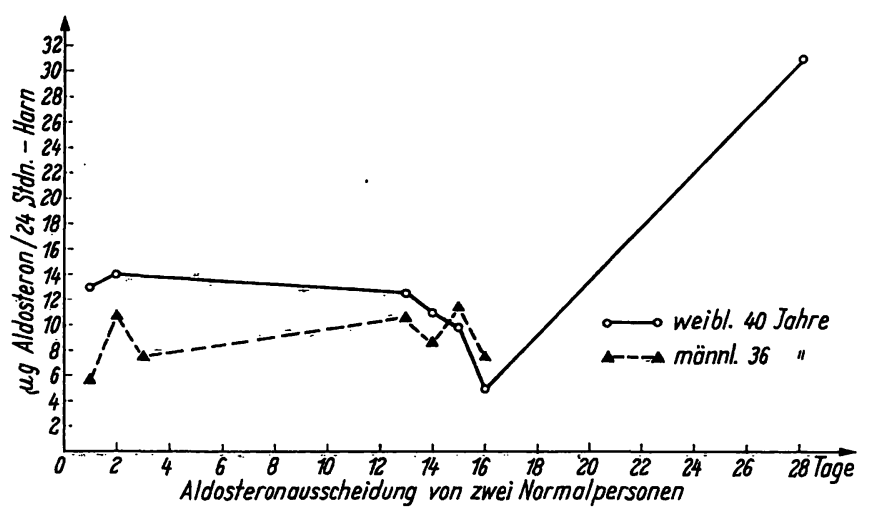

Abb. 1

Tab. 4

Ergebnisse dex Aldosteronbestimmungen von 6 pathologischen Harnen und einem Schwangerschaftsharn

\begin{tabular}{lccll}
\hline Name & Alter & $\begin{array}{c}\text { Harnmenge } \\
\mathrm{m} / / 24 \text { Stdn. }\end{array}$ & $\begin{array}{c}\text { Aldosteron } \\
\mu \mathrm{g} / 24 \text { Stdn. }\end{array}$ & Klinische Diagnose \\
\hline E. S. & 40 & 625 & 40 & Cyclischer Hyperaldosteronismus \\
K. H. & 16 & 650 & 17 & Conn-Syndrom \\
G. T. & 45 & 880 & 36 & Dekompensiertes Herzvitium \\
L.S. & 61 & 1500 & 49 & Lebercirthose \\
I. M. & 46 & 650 & 4 & Addison \\
W. H. & 59 & 1400 & 26 & Cisbing \\
B.S. & 20 & 1147 & 31 & Schwangerschaft (3. Monat) \\
\hline
\end{tabular}

Tab. 5

Normalwerte, Schwankungsbreite und Wiederfindungsversuche von Aldosteron im Harn bei verschiedenen Autoren

\begin{tabular}{|c|c|c|c|c|c|}
\hline Autor & $\begin{array}{l}\text { Zahl der } \\
\text { Personen }\end{array}$ & Geschlecht & $\begin{array}{l}\mu \mathrm{g} \text { Aldosterc } \\
\text { Mittelwert }\end{array}$ & $\begin{array}{l}\text { n/24-Stdn.-Harn } \\
\text { Schwankungs- } \\
\text { breite }\end{array}$ & $\begin{array}{c}\text { Wieder- } \\
\text { findung } \\
\%\end{array}$ \\
\hline $\begin{array}{l}\text { NeHER, WeTtSTEIN } \\
\text { (1956) }\end{array}$ & - & - & $(4,5)$ & $1,0-9,0$ & 75 \\
\hline \multirow[t]{2}{*}{ MOOLENAar (1957) } & 9 & männl. & 8,0 & $5,0-13,0$ & - \\
\hline & 12 & weibl. & 4,5 & $2,0-8,0$ & - \\
\hline AYRES (1957) & 31 & männl. & 11,0 & $4,6-23,5$ & 77 \\
\hline NoWACZYNSKI (1957) & 9 & - & 5,1 & $2,2-10,0$ & 83 \\
\hline HERnando (1957) & 17 & - & 5,0 & $0,0-15,0$ & $20-31$ \\
\hline $\begin{array}{l}\text { BaULIEU, DE VIGAN } \\
\text { (1958) }\end{array}$ & - & - & 3,0 & $1,0-6,0$ & 75 \\
\hline RoMANI (1958) & 5 & - & - & $2,2-10,4$ & - \\
\hline DyRENFURTh, VenNING & 5 & männl. & - & $1,4-9,1$ & 60 \\
\hline (1959) & 7 & weibl. & - & $1,1-7,0$ & - \\
\hline Brooks (1960) & 4 & - & 5,6 & $4,8-6,3$ & 70 \\
\hline $\begin{array}{l}\text { KLiman, Peterson } \\
\quad(1960)\end{array}$ & 26 & - & 10,0 & $5,0-19,0$ & 105 \\
\hline BrombaCHER, HARTING & 5 & männnl. & - & $6,3-11,0$ & - \\
\hline$(1961)$ & 4 & weibl. & - & $7,6-9,8$ & - \\
\hline $\begin{array}{l}\text { NishikAZE, STAUDINGER } \\
\text { (1962) }\end{array}$ & - & 一。 & 10,0 & 一 & - \\
\hline Gerdes, Staib (1965) & .10 & - & 8,8 & $3,6-17,8$ & 70 \\
\hline $\begin{array}{l}\text { NowOtNY, StAUDINGER } \\
\text { (1965) }\end{array}$ & 20 & - & 12,4 & $3,0-20,0$ & 80 \\
\hline StaIb (1961) & 30 & - & - & $3,0-16,0$ & 80 \\
\hline \multirow[t]{2}{*}{ SIEGENTHALER (1962) } & 6 & männl. & 9 & $4,0-16,0$ & 74 \\
\hline & 4 & weibl. & 13 & $7,0-15,0$ & - \\
\hline
\end{tabular}




\section{Patbologischer Harn}

In Tabelle 4 sind die Ergebnisse von 6 pathologischen und einem Schwangeren-Harn zusammengefaßt; sie stimmen mit denen von anderen Autoren $(21,23)$ gut überein. Die Werte, die wir bei einem klinisch diagnostizierten Fall von Conn-Syndrom gefunden haben, liegen im Bereich der Norm. Es ist daran zu denken, daß hier die Ausscheidung von Tetrahydroaldosteron erhöht sein könnte, was mit unserer Methode nicht zu erfassen ist. Auf diese Möglichkeit einer erhöhten Ausscheidung von Aldosteronmetaboliten beim Conn-Syndrom hat schon NeHER (23) aufmerksam gemacht.

\section{Diskussion}

Das jetzt von uns beschriebene Verfahren zur Bestimmung des Aldosterons im Harn zeichnet sich dadurch aus, daß der Harnextrakt nach säulenchromatographischer Vorreinigung durch nur eine zweidimensionale Dünnschichtchromatographie getrennt wird. Das bedeutet gegenüber' anderen Verfahren einen erheblichen Zeitgewinn.

Das nach der zweidimensionalen Dünnschichtchromatographie isolierte Aldosteron ist weitgehend rein, wie die zweidimensionale Rechromatographie mit anderen Lösungsmittelsystemen und der Vergleich mit authentischem Aldosteron zeigte. Auch die Fluoreszenz- und Absorptionsspektren der Schwefelsäurechromogene von dem aus Harn isolierten Aldosteron sind identisch mit den Spektren von reinem Aldosteron. Die Spezifität unserer Methode beruht auf der Kombination einer zweidimensionalen Dünnschichtchromatographie mit der für $\Delta^{4}$-3-Ketosteroide spezifischen Fluoreszenz auf einer Lithiumhydroxyd-Oberfläche.

Das Verfahren liefert gute Resultate. Das erkennt man, wenn man die nach unserer Methode ermittelten Normalwerte mit denen anderer Autoren vergleicht. Wie aus Tabelle 5 ersichtlich ist, liegt das Mittel der Normalwerte bei uns um ein Geringes höher als die der meisten anderen Autoren. Allerdings sind auch die Wiederfindungen bei diesen Methoden meistens geringer als bei unserer Methode. Dagegen stimmen unser Mittelwert und die Schwankungsbreite gut mit denen, die mit der Isotopenverdünnungsmethode (7) gefunden wurden, überein.

$\mathrm{Da}$ die Methode spezifisch und genügend genau ist, dabei nur $1 / 10$ Volumen des Tagesharns benötigt und außerdem methodisch weniger aufwendig ist, als die bisher beschriebenen, glauben wir, daß sie sich als Routinemethode eignet.

Wir danken Fräulein Margor Binger für geschickte Mitarbcit. Herrn Dr. R. Neher, Fa. CIBA AG. Basel/Schweiz, danken wir für die freundlich überlassene Probe Aldosteron.

Der Alexander-von-Humboldt-Stiftung dankt der cine von uns - EDGAR NowOTNY - für ein Stipendium; dem „Fonds der Chemie“ danken wir für Unterstützung der Arbeiten.

\section{Literatur}

1. Neher, R. und A. Wettstein, Acta endocr. K'hvn 18, 386 (1955). - 2. NeHer, R. und A. Wettstein, J. Clin. Invest. 35, 800 (1956). - 3. MoolenaAr, A. J., Acta endocr., K'hvn 25, 161 (1957). - 4. Ayres, P. J., O. Garrod, S. A. Smmpson und J. F. Tait, Biochem. J. 65, 639 (1957). - 5. Novaczynskr, W. J., R. P. Steyermark, E. Korw, J. Genest und R. N. Jones, Canad. J. Biochem. Physiol. 34, 1923 (1956). - 6. NowaczYNski, W. J., E. E. Korw und J. Genest, Canad. J. Biochem. Physiol. 35, 425 (1957). - 7. Kliman, B. und R. E. Peterson, J. biol. Chemistry 235, 1639 (1960). - 8. Staib, M. C., J. F. Dingman und K. W. Fester, J. Clin. Endocr., Springfield 21, 148 (1961). - 9. Siegenthaler, W. E., A. Dowdy und J. A. Luetscher, J. Clin. Endocr., Springfield 22, 172 (1962). - 10. Hernando, L., J. Crabbé, E. J. Ross, W. J. REDDy, A. E. Renold, D. H. Nelson und G. W. Thorn, Metabolism, Baltimore 6, 518 (1957). - 11. Baulieu, E. E. und M. DE VIGAN, Rev. franç. Études clin. biol. 3, 71 (1958). - 12. RoMANI, J.D., Presse méd., (Paris) 66, 837 (1958). - 13. Dyrenfurth, I. und E. H. Venning, Endocrinology 64, 648 (1959). - 14.
Broocks, R. V., Mem. Soc. Endocr. 8,9 (1960). - 15. BromBACHER, P. J. und M. C. Harting, Clin. chim. Acta (Amsterdam) 6, 886 (1961). - 16. Gerdes, H. und W. Starb, Klin. Wschr. 43, 789 (1965). - 17. Nishikaze, O. und HJ. Staudinger, Klin. Wschr. 40, 1014 (1962). - 18. Nowotny, E., R. Abraham und HJ. Staudinger, diese Z. 3, 8 (1965). - 19. Abraham, R. und HJ. Staudinger, $Z$. Naturforsch. $18 b, 421$ (1963). - 20. Abraham, R. und HJ. Staudinger, diese Z. 2, 16 (1964). - 21. Stark, G., zit. in: Aldosteron, 9. Symposium der Dtsch. Ges f. Endokrinologie. Wiesbaden, Mainz, 3.-5. Mai 1962, Springer-Verlag, BerlinGöttingen-Heidelberg (1963). - 22. Sims, E. A. H., C. I. MeEKER, M. J. Gray, M. Watanabe und S. Solomon, zit. in: Aldosteron A Symposium Organized by the Council for Internat. Organiz. of Med. Sc., established under the Joint Auspices of UNESCO \& wHo., Blackwell Scientific Publications, Oxford (1964). - 23. NeHER, R., zit. in: Aldosteron, S. 24, Tab. 2. 9. Symposium der Dtsch. Ges. f. Endokrinologie. Wiesbaden, Mainz 3.-5. Mai 1962, SpringerVerlag, Berlin-Göttingen-Heidelberg (1963).
Professor Dr. Hj. Staudinger 63 Gießen,

Friedrichstr. 24 